Case Report

OPen Access

CrossMark

\title{
High-grade testicular diffuse $B$ lymphoma, a rare localization: a case report and review of the literature
}

\begin{abstract}
Testicular Diffuse lymphoma B with large cells is a rare subgroup of testicular tumors. The invasion may be synchronous or metachronous, unilateral or bilateral. The therapeutic attitude depends imperatively on the stage of the disease and determines the prognosis. We report the different epidemiological, clinical and therapeutic aspects of this type of cancer and a review of the literature through a rare observation of a primary testicular diffuse lymphoma B with non-centro-follicular cells in a patient of 66 years treated with chemotherapy followed by radiotherapy in the testicles. The post-therapeutic results were in favor of complete remission.
\end{abstract}

Keywords: diffuse lymphoma B, testicles, chemotherapy, radiotherapy
Volume II Issue I - 2020

\author{
Zakaria Ahmed Youbi, ,,2 Sena Yossi,, ${ }^{2}$ Pierre \\ Sesques, ${ }^{2}$ Hassan Jouhadi' \\ 'Department of Radiation therapy, Mohamed VI Center for \\ cancer treatment, Morocco \\ 2Department of Radiation therapy, Hospital Center, Lyon Sud, \\ France
}

Correspondence: Zakaria Ahmed Youbi, Department of Radiation therapy, Mohamed VI Center for cancer treatment, CHU Ibn Rochd, Casablanca, Morocco, Tel 00212662029092, Email zakara.youbi@gmail.com

\section{Introduction}

Primary testicular lymphoma is a rare entity that accounts for 1 to $7 \%$ of all malignant tumors of the testis ${ }^{1,2}$ and $1 \%$ of non-Hodgkin's lymphoma (LMNH). It mainly affects men over 60 years of age. ${ }^{3}$ Its prognosis is often reserved and marked by the frequency of recurrences. Management depends strictly on the stage of the disease.

\section{Observation}

Mr O.A, aged 66 years old, followed for high blood pressure under medical treatment who consulted for lumbar pain associated with a bilateral testicular tumefaction and maxillary mass, rapidly increasing in volume. Clinical examination found:

a. Bilateral testicular swelling with inguinal adenopathies.

b. Right maxillary tumor mass associated with homolateral cervical adenopathy.

CT scan objective bilateral testicular lesions associated with bilateral inguinal lymphadenopathy and mass syndrome centered on the lower right maxillary sinus with large necrotic adenopathy of the right $1 \mathrm{~B}$ zone. The largest one measured $47 \times 36 \mathrm{~mm}$, and multiple adenopathies of the two-supraclavicular hollows (Figure 1).

Positron emission tomography (PET-TDM FDG) showed bilateral testicular lymphomatous involvement with a maximum SUV evaluated to 18.42 on the right side and 16.72 on the left side, the right testis measured $44.3 \times 36.9 \mathrm{~mm}$, a supra and infra-diaphragmatic lymph node involvement with invasion of right submaxillary, amygdala, maxillary sinus and underlying bone structures, associated with bilateral renal locations (Figure 2).

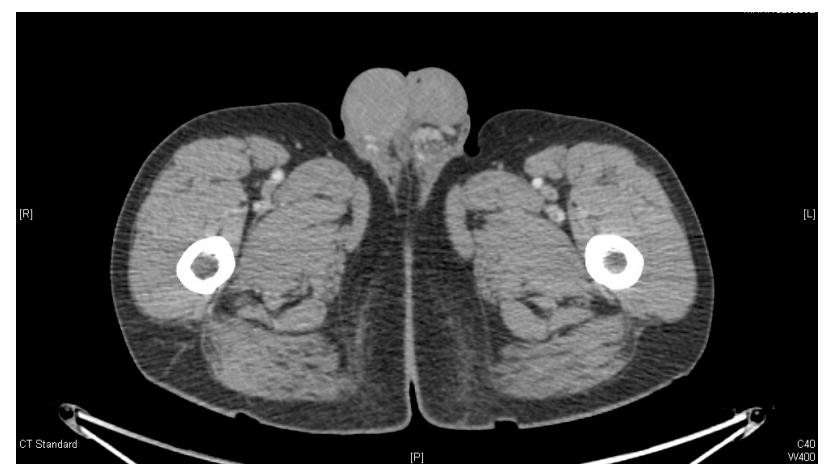

Figure I Pelvic CT in axial section showing testicles increased in volume with a tumor appearance.

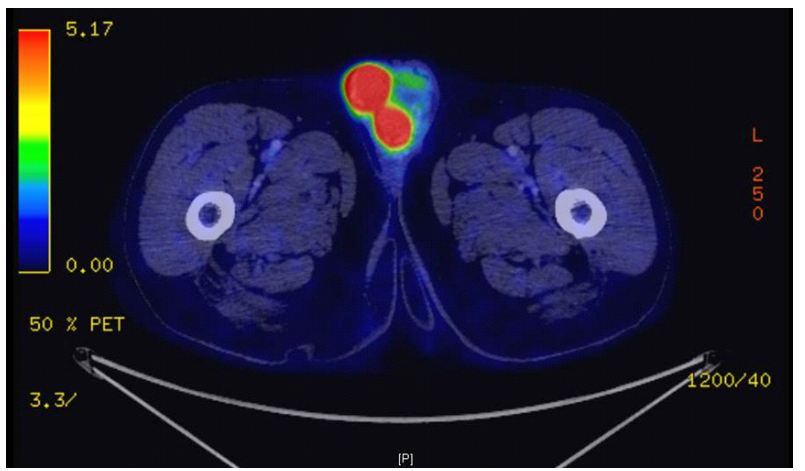

Figure 2 FDG PET-CT: Bilateral testicular lymphoma with a SUV max at I8.42 on the right side and 16.72 on the left side. 
Biopsies were realized. Anatomopathologic and immunohistochemical study demonstrated a large-cell diffuse lymphoma with a non-centro-follicular phenotype (Ki67 at 60\%, CD20 positive, C-MYC negative/BCL2 positive/BCL2 positive / BCL6 positive). Biologically, tumor markers (Alpha foeto-protein and $\beta$ HCG) were normal. The patient had 8 cycles of immunochemotherapy type R-CHOP and METHOTREXATE high dose. FDG-PET scan at the end of chemotherapy showed a very good response on all targets. The dossier was discussed at a multidisciplinary consultation meeting, which retained the indication of radiotherapy (RT) at the dose of $30 \mathrm{~Gy}$ in 15 fractions of $2 \mathrm{~Gy}$ in $3 \mathrm{D}$ conformal technique (Figure 3). Control FDG-PET scan, one month after the end of the RT, was in favor of complete remission according to CHESON 14 criteria. Consequently, the patient will be placed under surveillance at the rate of a clinical consultation every 3 months for 2 years associated with a biological check-up and then every 6months up to 5years.

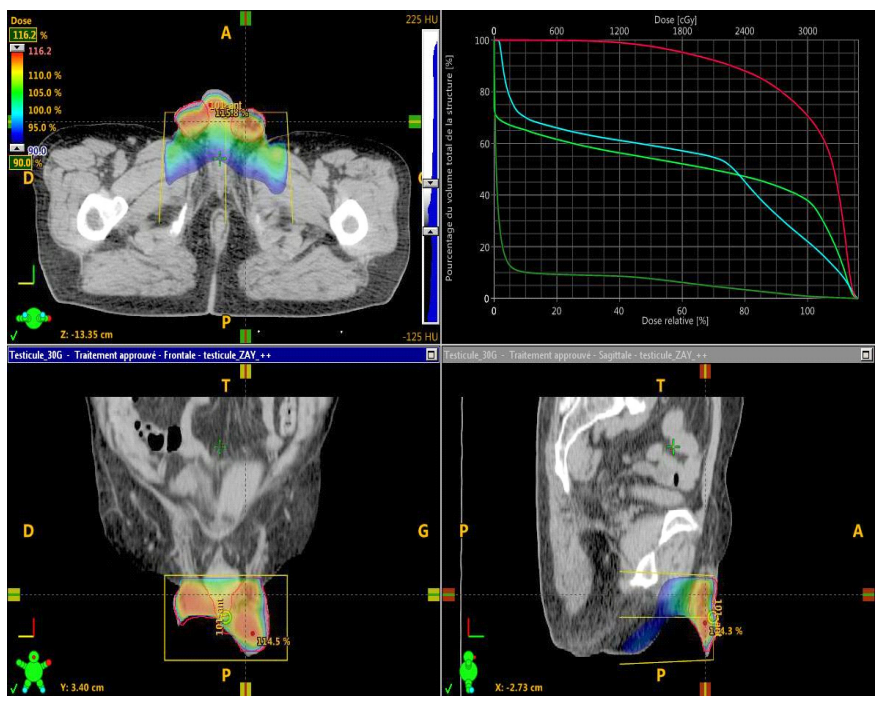

Figure 3 Three-dimensional 3D conformal radiotherapy using a direct beam of 6 Mev energy photons.

\section{Discussion}

Although the primary testicular location of diffuse B lymphoma is rare and generally occurs in the 5 th decade (beyond 50 years), it accounts for less than $1 \%$ of non-Hodgkin's lymphoma. ${ }^{1}$ The disease is often unilateral but also bilateral in $18 \%$ of cases. ${ }^{1-3}$

The most frequent clinical picture was a painful testicular mass, unilateral or bilateral, identified during the initial or systemic assessment of malignant lymphoma or in the clinical follow-up of patients with lymphoma. ${ }^{3}$ The other clinical signs that can be associated are hydrocele (in $40 \%$ of cases), fever, night sweats, anorexia and weight loss (in $25-41 \%$ of cases). ${ }^{3-5}$ Historically, primary testicular lymphoma is correlated with a poor prognosis with a 5-year overall survival rate of $17-48 \%$, especially non-centrofollicular large cell diffuse B lymphoma, and a strong metastatic Central nervous system and skin, whose mechanisms are not yet elucidated. ${ }^{1-6}$ On the paraclinic plane, bilateral testicular ultrasound shows a focal hypoechogenic mass with no capsular involvement or diffuse hypoechogenic lesion of the whole testis in contrast to hyperechogenic tissue. ${ }^{4}$ The extension report currently consists of a position-emitting tomography (FDG PET scan) in search of other extra-ganglionic lymphomatous sites, including the Waldeyer ring, skin, lungs and central nervous system. $., 7,8$

Anatomopathology and immunohistochemical examination is essential to make a diagnosis of certainty showing the expression of CD19, CD20, CD22, CD45, CD10 cell biomarkers (found in 30\% to $60 \%$ of cases) and the MUM-1 transitional marker (Found in 35\% to $65 \%$ of diffuse B lymphomas). ${ }^{9-12}$ The Ki67 proliferation index is often higher than $40 \%{ }^{13,14}$

The cytogenetic study and in situ fluorescent hybridization demonstrated the existence of complex genetic alterations (translocations, amplifications, and deletions) in diffuse B lymphomas with large non-follicular testicular cells, most commonly the chromosomal abnormalities $3 \mathrm{q} 27$ and deletions of $6 \mathrm{q} .{ }^{15}$

Treatment depends on the stage of the disease according to the American Joint Committee on Cancer (AJCC) classification, 7th edition: localized (stage I/II) or advanced (III/IV) ${ }^{4-16}$ For localized stages, the therapeutic standard is not yet established. Diagnostic and therapeutic orchidectomy is the preferred treatment since the blood-testicular barrier makes testicular tumors inaccessible to systemic treatments. ${ }^{4}$ Cyclophosphamide, doxorubicin, Vincristine and Prednisone (CHOP) was the pillar of chemotherapy protocols for several years. More recently, the addition of Rituximab, which is an anti-CD20 monoclonal antibody to chemotherapy (R-CHOP), has significantly improved progression-free survival and overall survival. ${ }^{14}$ The relapse in the central nervous system and frequent hence the interest of prophylactic intrathecal chemotherapy. Radiotherapy for advanced stages can be used prophylactically to prevent recurrence in the regional lymph nodes and the controlateral testis, but also to treat lymphomatous lesions such as retroperineal nodules. ${ }^{4-14}$ For advanced stages (III/IV), the therapeutic standard corresponds to the guidelines, was based on an anthracycline chemotherapy associated with Rituximab, testicular and scrotal radiotherapy for closing at the dose of $30 \mathrm{~Gy}$ in 15 fractions of $2 \mathrm{~Gy}$, and intrathecal chemotherapy. ${ }^{4}$

The standard treatment for recurrences is not yet defined. Patients should be treated according to the therapeutic strategy of aggressive forms of malignant non-Hodgkin's lymphoma and influenced by patient age, performance status (PS), and treatment-related toxicity. ${ }^{4-14}$ Immunotherapy is currently taking up more and more space in the second line metastatic therapeutic arsenal after cisplatin refraction and constitutes a promising avenue of research which may change the prognosis of this disease in the future. ${ }^{16,17}$

Testicular B-cell diffuse lymphoma with large non central follicular cells remains an aggressive disease with a maximum of recurrences during the first 2 years of follow-up. In the largest group of patients studied by The International Extranodal Lymphoma Study Group, survival at age 5 and 10 was $48 \%$ and $27 \%$, respectively. ${ }^{8}$ The different prognostic factors reported in this study were age, performance status (PS), systemic symptoms, tumor size $>9 \mathrm{~cm}$, invasion of the spermatic cord, serum lactate dehydrogenase, Histological grade, vascular invasion and tumor stage. ${ }^{1-14}$

\section{Conclusion}

Primary testicular involvement remains a relatively rare localization of diffuse B lymphoma, requiring an accurate diagnosis for better management. Treatment remains complex, depending on 
the stage of the disease. Chemotherapy and radiotherapy are the main therapeutic weapons. Despite a well-conducted treatment, the risk of recurrence is important making the prognosis a little reserved.

\section{Authors contribution}

All authors contributed to the writing of this manuscript according to the criteria of the ICMJE. All authors have read and approved the final version of the manuscript.

\section{Acknowledgments}

I thank Dr. Sena YOSSI for his kindness and helpful contribution to the writing of this article.

\section{Conflicts of interest}

The authors do not declare any conflict of interest.

\section{References}

1. Zucca E, Conconi A, Mughal TI, et al. Patterns of outcome and prognostic factors in primary large-cell lymphoma of the testis in a survey by the International Extranodal Lymphoma Study Group. J Clin Oncol. 2003;21(1):20-27.

2. Zucca E, Roggero E, Bertoni F, et al. Primary extranodal non-Hodgkin's lymphomas, part 1: gastrointestinal, cutaneous and genitourinary lymphomas. Ann Oncol. 1997;8(8):727-737.

3. Shahab N, Doll DC. Testicular lymphoma. Semin Oncol. 1999;26(3):259269.

4. Vitolo U, Ferreri AJ, Zucca E. Primary testicular lymphoma. Crit Rev Oncol Hematol. 2008;65(2):183-189.

5. Moller MB, d'Amore F, Christensen BE. Testicular lymphoma: a population based study of incidence, clinicopathological correlations and prognosis. The Danish Lymphoma Study Group, LYFO. Eur J Cancer. 1994;30A(12):1760-1764.

6. Li D, Xie P, Mi C. Primary testicular diffuse large B-cell lymphoma shows an activated B-cell-like phenotype. Pathol Res Pract. 2010;206(9):611615 .
7. Fonseca R, Habermann TM, Colgan JP, et al. Testicular lymphoma is associated with a high incidence of extranodal recurrence. Cancer. 2000;88(1):154-161.

8. Swerdlow SH, Campo E, Harris NL, et al. Pathology and Genetics of Tumours of Hematopoietic and Lymphoid Tissues. World Health Organization Classification of Tumors. Lyon, France: IARC Press; 2008.

9. Loddenkemper C, Anagnostopoulos I, Hummel M, et al. Differential Emu enhancer activity and expression of BOB.1/OBF.1, Oct2, PU.1, and immunoglobulin in reactive B-cell populations, B-cell non-Hodgkin lymphomas, and Hodgkin lymphomas. Pathol. 2004;202(1):60-69.

10. Berglund M, Thunberg U, Amini RM, et al. Evaluation of immunophenotype in diffuse large B-cell lymphoma and its impact on prognosis. Mod Pathol. 2005;18(8):1113-1120.

11. Colomo L, Lopez-Guillermo A, Perales M, et al. Clinical impact of the differentiation profile assessed by immunophenotyping in patients with diffuse large B-cell lymphoma. Blood. 2003;101(1):78-84.

12. Miller TP, Grogan TM, Dahlberg S, et al. Prognostic significance of the Ki 67 associated proliferative antigen in aggressive non-Hodgkin's lymphomas: a prospective Southwest Oncology Group trial. Blood. 1994;83(6):1460-1466.

13. Matthew JH, Adebowale J. Primary Diffuse Large B-Cell Lymphoma of the Testis. Arch Pathol Lab Med. 2011;135(10):1363-1367.

14. Bosga-Bouwer AG, Kok K, Booman M, et al. Array comparative genomic hybridization reveals a very high frequency of deletions of the long arm of chromosome 6 in testicular lymphoma. Genes Chromosomes Cancer. 2006; 45(10):976-981.

15. Edge SB, Byrd DR, Compton CC, et al. AJCC Cancer Staging Manual. 7th ed. New York: Springer; 2010.

16. Andrea N, Gennady B, Robert JC, et al. Genomic characterization of testicular germ cell tumors relapsing after chemotherapy. Eur Urol Focus. 2020;6(1):122-130.

17. Lakshmi N, Fabio MI, Ann LC, et al. PD-1 blockade with nivolumab in relapsed/refractory primary central system and testicular lymphoma. Blood. 2017;129(23):3071-3073. 